\title{
The Role of Nitric Oxide in the Integrity of Endothelial Glycocalyx
}

\author{
R.F. Neamu, S. Predescu, T. Voyno-Yasenetskaya, A. B. Malik and D. Predescu \\ Department of Pharmacology, University of Illinois at Chicago, $835 \mathrm{~S}$ Wolcott Ave, Chicago, IL, \\ 60612, United States of America
}

Endothelial cells rely on $\mathrm{NO}$ as an autocrine and paracrine factor for the regulation of their functions. Our previous studies evaluating the effects of absence or excessive production of endothelial nitric oxide synthase (eNOS)-derived NO upon the morphology and function of endothelial barrier [1,2] clearly demonstrated that physiological amounts of NO are mandatory for a normal basal permeability. The systematic examination of endothelial barrier, under abovementioned conditions, proved that the interendothelial space is the main passageway affected. However we have recorded other morphological changes of the endothelia, and one of the most constant variation encountered was the alteration of the endothelial glycocalyx. The endothelial glycocalyx, a negatively charged mesh of membrane-attached glycoproteins, proteoglycans, glycosaminoglycans and adsorbed plasma proteins, has been recognized to have multifaceted physiological functions and to be a determinant of endothelial dysfunction. One of the functions credited to the glycocalyx is its role as a molecular sieve able to determine the oncotic forces across the endothelial barrier and maybe to justify the sharp break in the solute permeability for molecule with diameters larger than $4 \mathrm{~nm}$.

Published studies have unveiled the interrelationship between the glycocalyx and NO production. One study showed that the removal of the hyaluronidan (a glycocalyx component) after the treatment with hyaluronidase led to a decreased NO production [2]. In another study, ischemiareperfusion injury in guinea-pig hearts induced endothelium glycocalyx disruption. In this case, NO had a detrimental effect, because its reaction with superoxide generated peroxynitrite, a toxic product, that led to the abnormalities in the glycocalyx [3]. Based on these observations we hypothesized that variations in the plasma levels of NO can lead to alterations of endothelial glycocalyx.

It was shown before that eNOS knock-out mouse has decreased levels of plasma NO compared to wt mouse, while the absence of caveolin-1 (cav-1) gene leads to an up elevation in eNOS activity and increased plasma NO levels in the cav-1 knock-out mice $[1,5]$. In our present experiments we perfused the vascular beds from different organs from wild-type (wt), cav-1 knock-out and eNOS knock-out mice with fixative mixtures containing one of the following three stains: lanthanum chloride, alcian blue 8GX, and ruthenium red. These stains are considered "positive stains" for electron microscopy due to increased electron density and their usefulness in analyzing the morphology of endothelial glycocalyx is well documented [6]. After perfusion, the organs were removed, minced in small pieces, and selected specimens further processed for analysis in the transmission electron microscope.

We examined the endothelial glycocalyx in the myocardium, diaphragm, and aorta of the abovementioned mice. Qualitatively, we observed a marked reduction in the thickness of glycocalyx in both knockout mice in all the studied vascular beds with associated lack of continuity, large gaps 
and a general "patchy" appearance. In wt mice the glycocalyx appeared as a continuum, with a thickness between 50-100 nm.

Our results indicate that in the knock-mice modified plasma NO level has a profound effect on the morphology of the glycocalyx that could induce alterations in normal physiological events, such as leukocyte adhesion to endothelium or mechanotransduction.

\section{References}

[1] D. Predescu, S. Predescu, J. Shimizu, K. Miyawaki-Shimizu, A.B. Malik, Am J Physiol Lung Cell Mol Physiol. 2005 Sep;289(3):L371-81.

[2] K. Miyawaki-Shimizu, D. Predescu, J. Shimizu, M. Broman, S. Predescu, A.B. Malik. Am J Physiol Lung Cell Mol Physiol. 2006 Feb;290(2):L405-13. Epub 2005 Sep 23.

[3] S. Mochizuki, H. Vink, O. Hiramatsu. T. Kajita, F. Shigeto, J. A. E. Spaan, F. Kajiya, Am J Physiol Heart Circ Physiol. 2003 Aug;285(2):H722-6

[4] M. Kurzelewski, E. Czarnowska, A. Beresewicz, J Physiol Pharmacol. 2005 Jun;56(2):163-78. [5] YY. Zhao, Y. Liu, R.V. Stan, L. Fan, Y. Gu, N. Dalton, P.H. Chu, K. Peterson, J. Ross Jr, K.R. Chien, Proc Natl Acad Sci U S A. 2002 Aug 20;99(17):11375-80. Epub 2002 Aug 12.

[6] M. A. Hayat, Positive staining for electron microscopy, Van Nostrand Reinhold Co., New York, 1975

[7] This work was supported by the National Institutes of Health grant 4942 P01HL060678-06 Proj. 3 to T. Voyno-Yasenetskaya and A.B. Malik

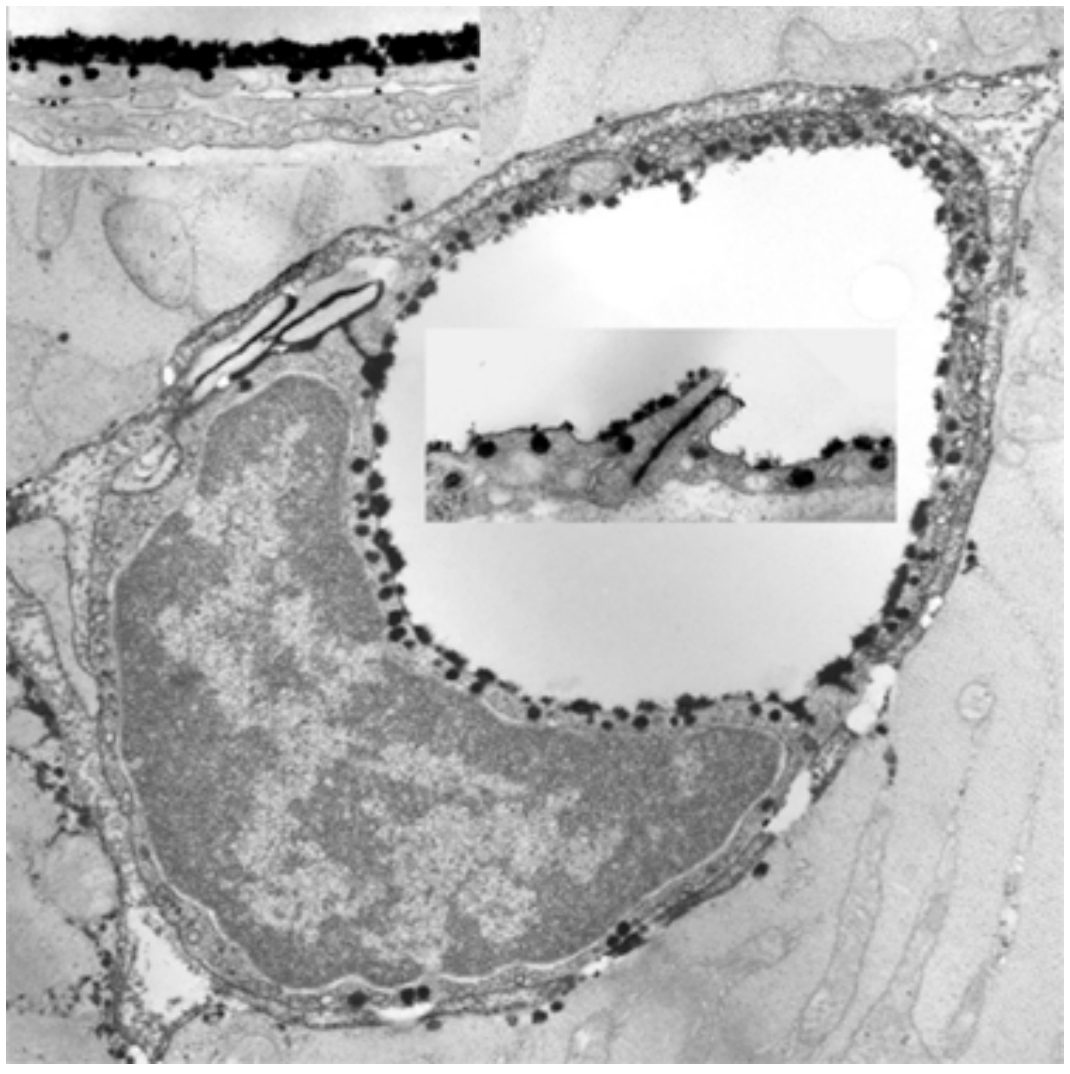

Representative electronmicrograph depicting the morphology of endothelial glycocalyx in the capillary of an eNOS knock-out mouse after perfusion with a fixative containing lanthanum chloride. The upper inset is a representative staining of endothelial glycocalyx, seen as a continuum, in a wt mouse. The lower inset is a higher magnification image of the endothelial glycocalyx in eNOS knock-out mouse, showing profound lack of continuity in the glycocalyx 